Auszug aus der Dissertation über:

\title{
Die Entwicklung des deutschen Klavierkonzertes von Mozart bis Liszt
}

von $\mathrm{HANS}$ FNGEL.

Referent: Geheimrat Prof. Dr. ADOLF SANDBERGER.

Dekan: Professor Dr. HERBIG.

'Tag der mündlichen Prüfung: 7. Mäız 1925.

Zum Druck genehmigt: 9. April 1926.

Professor L. SCHERMAN, Dekan.

Das Wesen des Konzertes besteht in der Gegenüberstellung zweier Klangkörper, dem Solo und dem Tutti, die aus dem Concerto grosso erwachsen ist, das seinerseits wieder in der auch räumlich, durch die beiden Orgelemporen der Markuskirche gekennzeichneten Zweichörigkeit Willaerts, der Dialogtechnik des 16. Jahrhunderts und den älteren Ansätzen zur Zweichörigkeit in der niederländischen Polyphonie wurzelt. Zu diesem räumlichen Gegensatz der Klangkörper tritt im Sonatenkonzert der ganz anders geartete Gegensatz zweier Themen. Die Entwicklung des Klavierkonzertes von Mozart bis Liszt stellt sich dar als Kampf der beiden heterogenen Dualismen, dem Dialog der beiden Klangkörper und der $Z$ weithemigkeit der Sonatenform, für welche das Rondokonzert nicht ohne Bedeutung war. Im Anschluß an die Münchener Dissertation von Hugo Daffner „Die Entwicklung des Klavierkonzertes bis Mozart" (1906) wird in der Einleitung zunächst die Zeit vor Mozart betrachtet; bei einem Überblick über die Kouzerte der Schobert, Eichner, Giordani, Kellner, Beecke, Reichardt, Monn, Wagenseil, Steffan, Koželuch, Vanhall, Josef und Michael Haydn, Eichstaedt, Beckmann, Lang, Förster wird die Notwendigkeit betont, die Sonatenform als aus vielerlei Momenten erwachsenes allgemeines Gut, nicht als besondere Leistung einer "Schule“ zu betrachten. Die Scheidung zwischen „norddeutschem Cembalo-“ und
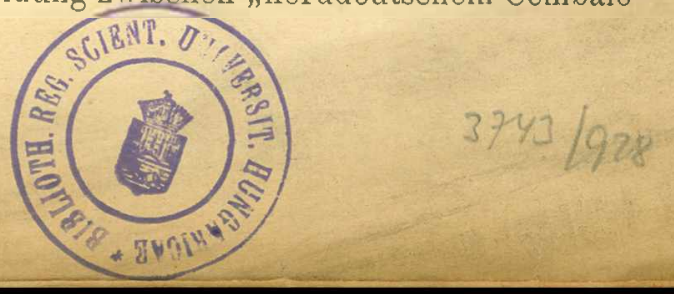
"süddeutschem Pianofortekonzert" ist nicht sachgemäß. Das „süddeutsche Pianofortekonzert" ist das Konzert in ausgeprägter Sonatenform im ersten und Rondo im letzten Satz. Cembalo, Clavichord und Klavier bestehen in beiden Lagern nebeneinander weiter, süddeutsche Komponisten mit modernem Pianofortestil schreiben häufig noch "Cembalo" und "Clavecin" auf die 'Titelblätter, in der Hochburg des „norddeutschen Cembalokonzertes" waren schon 1747 sieben Silbermannsche Flügel in Gebrauch. Nicht das Instrument hat den Stil bedingt und verbreitet, sondern umgekehrt; die musikalische Substanz und ihre Form ist das Primäre. Die konservative Haltung Berlins ist durch den persönlichen Geschmack Friedrichs des Großen bedingt; gerade der Führer der „Norddeutschen“, K. Pb. E. Bach, ist von großer Bedeutung für die Entwicklung der Sonatenform. Den uur bedingt geltenden Gegensatz von "norddeutschem Cembalokonzert“ und „süddeutschem Pianofortekonzert“ weiterhin verfolgen zu wollen, entspringt einer fälschlichen Gleichsetzung von „dramatisch-dialogisch“ mit ersterem und "solistisch-virtuos" mit letzterem. Die schematische Art, die Sonatentorm im Konzert anzuwenden, welche vor allem den ersten Satz betrifft, wird von den Zeitgenossen und Epigonen Mozarts geteilt. Mozart ist der einzige, der das Problem der Form erfaßt, der stets nach neuen Lösungen der Gegensätze beider Prinzipe sucht. Die Wiederholung des Vorderteiles der Sonatenform führt im Konzert zu einer an sich sinnlosen Zweiteilung in ein tonikabetontes Tutti und einen dominantbetonten Soloteil; am ehesten kommt die Durchführung dem Dialogeharakter des Konzertes entgegen; die Reprise bietet wieder dieselben Schwierigkeiten (Zusammenziehung des Tutti und des Soloteiles), wie überhaupt die Verteilung der Themen zwischen beide Klangkörper. Allerdings durchbricht Mozart nur einmal (in K.V.271) grundsätzlich die Tradition, indem er den Solisten sogleich zu Beginn teilnehmen läßt; es ist der erste Ansatz zur Vermeidung der stereotypen Zerteilung der Vorderteilwiederholungen im Sonatenkonzert, welche Beethoven, Mendelsohn, Schumann vollendeter glückte. Schier unerschöpflich sind Mozarts Einfälle bei der Verschmelzung von Dialogund Sonatenform, insbesondere dient ihm die Instrumentation zur Betonung des Dialogcharakters, besonders interessant sind die Durchführungen. Auch innerhalb seines eigenen Schaffens steht der Reichtum der Klangkombinationen, vor allem mittels der Bläser, einzig da, nirgends in seinem Schaffen finden sich sonst Effekte wie in den Mittelsätzen von K. V. 453,456, 482; ähnlich wie für Haydn die T'ätigkeit in der Esterhazyschen Kapelle, bedeuten Mozarts Akademien
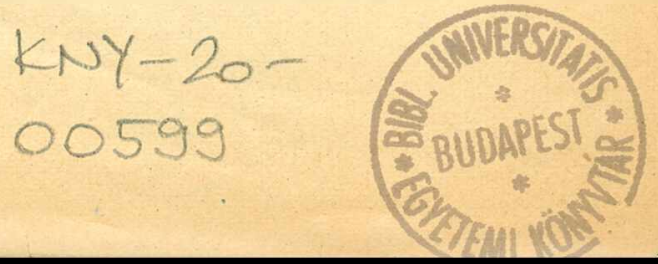
ein Experimentierfeld für seine Instrumentation. - Der Abstand Mozarts von seinen Zeitgenossen und Nachfolgern, die nur äußerlich seine Sprache nachahmen, in der Herausarbeitung des Dialogcharakters und der Heranziehung des Orchesters ist ungehener. Den Gegenpol zu Mozart bilden etwa Abt Voglers unbegleitete Konzerte, deren Gattung er "ganz neu" glaubt. Schon Bach und die Mitteldeutschen pflegten das unbegleitete Konzert, das noch bei Chopin, Liszt, Schumann erscheint, in dem „ad libitum"-Charakter des Orchesterparts der meisten „brillanten Konzerte“ fortlebte. Während das Registerspiel des Cembalo noch die Möglichkeit bot, Tutti und Soli klanglich auseinander zu halten, ist das "unbegleitete Konzert", ob sonatinenhaft im galanten Stil, oder Bravourstück der späteren Zeit, eine Sinnwidrigkeit. Vogel interessiert noch durch ein „Programm"konzert. Jagd- und Marschcharakter erscheint öfters, Lang bringt ein Pastoralkonzert. Nach Untersuchung der Konzerte von Sterkel, Cannabich, Stamitz, Dittersdorf, Rosetti, Schaden, Mederitsch, Pleyel, Gyrowetz, Hoffmeister, Beczwarskowsky, Maschek, Abeille, Schmidt, Schacht, Kospoth, Kerpen, Haueisen, Amon, Neubaur, Preindl, Freystädler, Destouches, Kanka, Mozart-Sohn, Wrantzky, Danzi, Müller, Schuster, Häßler wird zum „brillanten“ Konzert übergegangen. Louis Ferdinand, Himmel, Dussek, Wölfl, Steibelt, Cramer, Wilms, Klengel, Berger, Lessel, Schneider, Eberl, Kreutzer werden nach ibrer Bedeutung als Konzertkomponisten betrachtet. Das „brillante“ Konzert steht im Zeichen der sich rasch entwickelnden Technik. Das Musikalische tritt hinter der Erfindung von Spielfiguren zurück, auf welche größter Wert gelegt wird. Die "Spielepisoden“ überwuchern, der Orchesterpart verliert immer mehr an Bedeutung. Das Formproblem wird überhaupt vernachlässigt, man begnügt sich mit ganz oberflächlichem Schematismus. Im Gegensatz zu dem größten Zeitgenossen Beethoven, der in vieler Hinsicht unmittelbar an Mozart sich anlehnt (ein Einfluß des Konzertkomponisten K. Ph. E. Bach wird geleugnet), was in vielen Einzelheiten, Durchführung, Bläserbehandlung gezeigt wird. Die genialste Lösung des Konzertproblems bedeutet Beethovens G-Konzert. Beethovens Entwicklung wird rom Jugendkonzert bis ins Es-Konzert hinein, gelegentlich auch in einzelnen Spielfiguren, als zusammenhängend gezeigt. *) Die geistige und musikalische Vertiefung der Kunstgattung zeigt Beetboven in ähn-

*) Der Verfasser weist im „Beethoven-Jahrbuch“ Augsburg 1926, im Aufsatz "Der angeblich Beethovensche Klavierkonzertsatz" nach, daß dieser Satz der 1. Satz eines Klavierkonzertes vor J. J. Rösler ist. 
lichem Gegensatz zu seiner Umwelt, wie Mozart. - Das brillante Konzert ist gleichzeitig der Anfang des romantischen Konzertes. Die Entwicklung des romantischen Elementes bringt Steigerung der Klangbewertung (auch im Klaviersatz sich zeigend), Hinneigung zur reicheren und freieren Harmonik, Betonung der "Stimmung" im Gegensatz zur formalen Seite. „Romantik“ zeigt sich zunächst in den langsamen Sätzen; das Rondo behält den spielerischen Charakter. - Besondere Bedeutung wird Dussek bejgemessen. Dem Programmkonzert, wie das Steibelts, Wölfls, u. a. — "latente“ Programme sind häufig und typisch - steht das Concertino gegenüber, wie etwa in Kreutzers op. 50, das auch noch die beiden letzten Sätze mit einem Thema formt und schon im ersten Satz die Sonatenform so gut wie aufgibt. Zur Sonatenform hat die Romantik iberhaupt nur ein loses Verhältnis; sie verliert z. B. ganz das Verständnis für die Durchführung, an deren Stelle zunächst ein fremdes, meist sentimentales Thema ("lyrische Episode") erscheint, dem Spielfiguren folgen. Am schärfsten zeigt diesen Typ ein späteres Konzert der Romantik, Henselts Konzert f. Der Klangsinn der Romantik äußert sich in der Heranziehung eines zweiten Soloinstrumentes - bei den „Wienern“ war es die „spitze“ Oboe - nämlich des romantischen Cello im Mittelsatz (Lang, Rösler, Böhner, Weber, Mendelssohn, Klara Wieck, Schumann, Brahms). An musikalisch interessanten Werken fehlt es nicht, z. B. bringt Eberl im Konzert für zwei Klaviere einen Marsch als 2. Satz, der als Episode im letzten Satz wiederkehrt, beides Neuerungen. - Eine der fesselndsten Typen des romantischen Komponisten ist der genialische Ludwig Böhner, zugleich einer der wenigen, die Beethovens Einfluß zeigen. Erwähnenswert ist sowohl sein Versuch einer neuen Form (Concertino A), wie das mit einem Beethoven (Neunte) vorausnehmenden Baßrezitativ einsetzende Konzert op. 13 (1817). - Den Höhepunkt der romantischen Programmkonzerte bildet Webers Konzertstück op. 14. Zunächst dachte Weber an ein dreisätziges Abschiedskonzert (nach Beethovens Sonate "Les Adieux"). 1815 sind vier Sätze geplant das Programm wird detailiert, echt romantischerweise in die Zeit des deutschen Mittelalters zurïckgelegt. Es wird nachgewiesen, daß das Werk von Weber als dramatische (theatralische) Szene empfunden ist, wobei dem Klavier die Rolle des Subjektiv-Erlebenden (der Burgfrau im Programm) zufällt, das Orchester ganz im Sinne einer "Szene" schildert und begleitet. Damit ist das Konzertstück Vorgänger der ähnlich angelegten Konzerte von Spohr (,in Form einer 
Gesangsszene“) und Berlioz („Haraldsymphonie“). - Mit dem Konzertstïck Webers dringt das dramatische Element ins Konzert ein. In der Hinneigung zum Dramatischen liegt die wesentliche Steigerung, die das "Konzert der Virtuosen" gegenüber dem brillanten Konzert der Romantik bringt. Von letzterem werden noch Ries, Hummel, Czerny, Pixis, Zeuner, Clasing, Rummel, Schmitt, Arnold untersucht. Hauptrertreter des virtuosen Konzertes, das außer dem Dramatischen gekennzeichnet ist durch Pomp in der Technik (Oktaven, Akkorde im Gegensatz zur reinen Geläufigkeit des frühbrillanten Konzertes), Neigung zum Düster-Weltschmerzlichen, mit freilich oft nur salonmusikhafter äußerlicher Geste, sind Kalkbrenner, Chopin, dessen Verhältnis zu Hummel, Ries u. a. beleuchtet und dessen Bedeutung als Klavierkonzertkomponist skeptisch aufgefaßt wird, Herz, Thalberg, Henselt, Kullak, Mayer und vor allem J. Moscheles. Moscheles, zunächst unter dem Einfluß des „brillanten“ Konzertes, dann Beethovens, ist originell in der Art, wie er neue formale Elemente einführt, so das Rezitativ des Solos im 2. Konzert, das auch in seinem 3. Konzert (wie weiterhin bei Chopin, Liszt) erscheint, wohl auf Beethovens G-Konzert zurückgehend. Mit dem 6. Konzert erhebt er sich über die Durchschnittsvirtuosenkonzerte, beginnt er sich von der Sonatenform abzuwenden, mit der im romantischen Konzert (Steibelt) und später (Herz) geübten Anwendung eines halben Sonatensatzes. Bedeutsam ist die Verschmelzung von Tutti und Soli, das Arbeiten mit einem sich erst entwickelnden Thema, wie der Ansatz zu einer motivischen Satzverbindung. Den Höhepunkt in Moscheles Schaffen bedeutet das Concerto pathétique (Nr. 7), es bringt die bewußte Zurückstellung des PianistischVirtuosen gegenüber dem Inhaltlich-Musikalischen, wie eine neue und selbständige Behandlung der Form, auch die organische Satzverbindung durch das Motiv, wie sie später Liszt, durch Schuberts, von Liszt als Konzertstïck bearbeitete Wandrerphantasie angeregt, anwendet. Der Allegro agitato-Beginn des zweiten Satzes ist eine bedeutsame Anregung zur Einführung des Scherzos im Konzert, die der Moschelesschüler Litolff brachte. Moscheles Konzert, Meyerbees: gewidmet, jst auch bezeichnend für die seit dem Aufblühen der grolen Oper beginnende Beeinflussung der Klaviermusik durch diese. - Gleichzeitig mit der beginnenden Reform des Konzertes durch die großen Virtuosen, - auch diese waren fast durchwegs Deutsche - in den Weltplätzen Paris und London, beginnt in Deutschland eine Konzertreform durch Mendelssohn, der pianistisch kein Neuerer und Ërfinder, von der klassischen Musik ausgehend, vielleicht auch unter 
dem Eindruck altklassischer Werke, Tutti und Soli nicht mehr schematisch hintereinander setzt, sondern gleich zu Anfang in gemeinsamem Wettkampf beginnen läßt, überhaupt zu einer innigen Verknüpfung von Solo und Tutti gelangt. Weniger begründet und barmonisch als in Webers Konzertstïck, dessen Vorbild im Capriccio op. 22 von Mendelssohn noch stärker durchleuchtet, ist die Satzverbindung in Mendelssohns Konzert g. Taubert, Kufferath, der begabte und in Beethovens Spuren wandelnde Bennet (op. 7), Hiller, mit einem späten formal in der Verquickung von Tutti und Soli interessanten Konzert op. 69 fis, Frank, Klara Wieck, u. a.. müssen versinken neben der einzigen Teistung im Sonatenkonzert, die an genialer Lösung des Formproblems vielleicht noch über Beethovens G-Konzert gestellt zu werden verdient, Schumanns Konzert in a. Weitergehend als Mendelssohn gibt Schumann hier das übliche aus dem Kompromiß zwischen Konzert-Dialogcharakter und Sonatenform entstandene Formschema völlig auf, nicht aber die Sonatenform, die in inniger Verschmelzung von Solo und Tutti, die glücklichste Neugestaltung im Konzert findet. Iit der als Variation des Hauptthemas erkenntlichen As-Cantilene der Durchführung wird klassischer und romantischer Durchführungstyp ("Iyrische Episode") vereinigt. Feinste Zwiesprache beider Klangkörper herrscht im 2. Satz; Sonatenform im 3. Satz, der in der ganzen Literatur fast überall Rondoform zeigt. Die Reprise dieses Satzes ist merkwürdig, da sie (sonst nur einmal, bei Dittersdorf, im Konzert zu finden), die Exposition in der Unterdominante wiederholt. Auch Schumanns unterschätztes Konzertstück $G$, das die Sätze verbindet, zeigt eine ähnliche tonale Anlage von Exposition (e-C) und Reprise (e-G) im 1. Satz. Das Konzertstïck op. 139 weist eine seltsame Verkümmerung einer normalen Architektur auf, die psychologisch in Hinblick auf Schumanns Krankheit interessiert. - Große Bedeutung muß den Konzerten Litolffs zugewiesen werden, in denen die Sonatenform beibehalten, aber in steigendem Maße, immer großartiger zu einer eigentümlich „symphonischen" Verquickung des Solo, das bis dahin ungeahnt virtuos behandelt wird, und des genial instrumentierten Orchesters führt, in gewaltigsten Dimensionen, deren Bauprinzip sichtlich an Beethovens Durchführungen erwachsen ist. Daneben ist von größter Wichtigkeit die - vor einer vereinzelten Verwendung bei K. Ph. E. Bach abgesehen - hier erstmalige Einführung des Scherzos ins Konzert. Im 4. Konzert ist das Kräfteverhältnis zu Ungunsten des Klavieres verschoben, das oft als Instrument des Orchesters, freilich höchste Technik voraussetzend, behandelt wird. Genial ist Litolffs Architektonik 
die etwa mit einem Thema einen Satz von 400 Takten gestalten kann. In der wilden Dämonie des Scherzos wird Berlioz erkennbar, Liszt und Wagners Welt klingt voraus und an. Litolff steigert die Sonatenform in gigantische Ausmaße; Mittel dazu ist die häufig angewandte Technik der Durchführung (außerhalb des eigentlichen Durchführungsteiles). Klavieristisch überbietet das Concerto-Symphonique Litolffs, das dem Spieler oft mehr als das Übergewicht nimmt, an großzügiger Virtuosität, Klangfülle, Effekt, Brillanz, Pomp selbst den handlicheren Liszt. Der von Litolff nicht unbeeinflußte Liszt bricht im 1. Konzert endgültig mit der Sonatenform, an deren Stelle er vier (mit Scherzo) thematisch verbundene Sätze setzt; im A-Konzert wendet er eine Art Rondo-Variationsform an. Liszt gelingt die Verschmelzung beider dialogisch geführter Klanggruppen hervorragend; Konzertproblem und Form sind bei ihm geistreich und tief erfaßt. Leider steht seine allgemein künstlerische hobe Geistigkeit in argem Mißverhältnis zu seiner spezifisch-musikalischen Schöpferkraft.

Der durch die Entwicklung des Klavierkonzertes von Mozart bis Liszt gehende Kampf zwischen Konzertprinzip und Sonatenform führt bei Mozart, Beethoven, Mendelssohn, Schumann, Litolff zu Neuformungen im Sonatensinne, neben der schematischen Anwendung der Sonatenform über eine teilweise Aufgabe der Sonatenform (Concertino, Kreutzer, Moscheles u. a.) aber auch zur gänzlichen Aufgabe der Sonatenform (bei Liszt). Inhaltlich und auch klaviertechnisch geht die Entwicklung vom galanten Liebhaberkonzert (Wagenseil bis Vogler) über das brillante Gesellschaftskonzert (Dussek) zum Glanz des beginnenden Virtuosentums der Romantik (Weber) bis zum dramatischweltschmerzlich-dämonischen Pomp der großen Virtuosität (Kalkbrenner, Moscheles, Litolff, Liszt). 


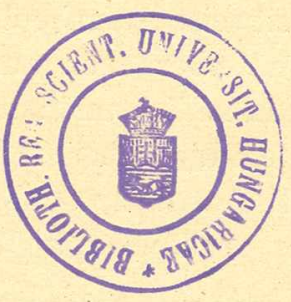

UNIVERSIT ̈̈TS-BUCHDRUCKEREI DR. C. WOLF\& SOHN, YÜNCHEN 